\title{
Topical Delivery System for Phytochemicals: Capsaicin and Capsicum Tincture
}

\author{
Bindu Thapa ${ }^{1}$, Ivan Pepic ${ }^{2}$, Zeljka Vanic ${ }^{2}$, Purusotam Basnet ${ }^{3,4}$ and Natasa Skalko-Basnet ${ }^{* 5}$ \\ ${ }^{1}$ The School of Pharmaceutical and Biomedical Sciences, Pokhara University, PO Box 427, Lekhnath, Nepal \\ ${ }^{2}$ Department of Pharmaceutics, Faculty of Pharmacy and Biochemistry, A. Kovacica 1, University of Zagreb, 10000 \\ Zagreb, Croatia \\ ${ }^{3}$ Women's Health and Perinatology Research Group, Department of Clinical Medicine, University of Tromso, \\ Tromso, Norway \\ ${ }^{4}$ IVF Unit, Department of Obstetrics and Gynaecology, University Hospital of North Norway, Tromso, Norway \\ ${ }^{5}$ Drug Transport and Delivery Research Group, Department of Pharmacy, University of Tromso, Universitetsveien \\ 57, N-9037 Tromso, Norway
}

*Corresponding author: Dr. Natasa Skalko-Basnet, Professor, Drug Transport and Delivery Research Group, Department of Pharmacy, University of Tromso, Universitetsveien 57, N-9037 Tromso, Norway, Tel: +47776 46 640, Fax: +47 77646 151, E-mail: natasa.skalko-basnet@uit.no

Citation: Bindu Thapa, Ivan Pepic, Zeljka Vanic, Purusotam Basnet, Natasa Skalko-Basnet (2013) Topical Delivery System For Phytochemicals: Capsaicin and Capsicum Tincture. J Pharm Drug Devel 1(2): 201. doi: 10.15744/2348-9782.1.201

Received Date: July 03, 2013 Accepted Date: September 19, 2013 Published Date: October 03, 2013

\begin{abstract}
Capsaicin, an active ingredient of Capsicum fruit, is currently undergoing "revival" in the clinical management of pain. However, the choice of its formulation is rather limited to the use of "old-fashioned" tinctures and recently the patches. In an attempt to improve the therapeutic outcome and develop its skin-friendly formulation, we prepared the vesicle-based drug delivery system with capsaicin. Moreover, the use of standardized Capsicum extract, rather than a single active ingredient, is proposed to lead to simplified and more cost-effective formulations. Phospholipid-based vesicles, namely liposomes and ethosomes, were prepared with capsaicin, Capsicum tincture or Capsicum powder and characterized for particle size, entrapment efficiency and stability. The vesicular dispersions were incorporated in the hydrogels to increase the formulation stability, its retention time at the skin and overall acceptability. Both the standardized crude Capsicum powder and Capsicum tincture were successfully employed as sources of capsaicin which is, to the best of our knowledge, reported for the first time. The reported phospholipid-based delivery system for capsaicin could represent an improved topical treatment of arthritic pain.
\end{abstract}

Keywords: Capsaicin; Phytochemicals; Liposomes; Ethosomes; Arthritis; Pain

\section{Introduction}

Although the use of herbal medicine has been increased all over the world, many of the promising phytochemicals suffer from limited bioavailability and their full potentials as medicine remains hampered by their physicochemical properties [1]. Capsaicin, the active principle found in Capsicum, and other TRPV1 agonists are currently undergoing "revival" in the clinical management of pain associated with inflammation, metabolic imbalances (diabetes), infections (HIV) and cancer [2-4]. Topical capsaicin should be considered as an adjunct to core therapy for knee and hand osteoarthritis according to the National Institute for Health and Clinical Excellence (July 2007) [5]. In addition, the widespread cultivation of Capsicum makes synthesis unnecessary, as large quantities of capsaicin can easily be extracted from readily available peppers [6]. What makes capsaicin unique among the natural irritants is that the initial excitation of sensory neurons is followed by a lasting refractory state, referred to as desensitization, when the previously excited neuron is unresponsive not only to capsaicin but also to diverse, unrelated stimuli. This desensitization is dose-dependent. Capsaicin was found to be very effective in reducing the pain associated with osteoarthritis, diabetic neuropathy, and psoriasis [4,7]. Topical drug application to treat arthritic pain is thought to deliver high local drug concentration with a reduced risk of systemic side effects. Biotransformation of capsaicin in human skin is slow and the majority of applied capsaicin remains unchanged with only a small fraction being metabolized to vanillylamine and vanillic acid. It was reported that maximal cutaneous capsaicinoid concentration was achieved quickly in the human stratum corneum and was both concentration and vehicle dependent, as was the elimination [8]. The topical capsaicin-containing analgesic products have an advantage of capsaicin residing at the local site of action unchanged over a longer period of time. Moreover, a lack of oxidative 
metabolism in the skin indicates that there is a lack of potential for formation of the covalently bound toxic metabolites [9].

However, the pungent and irritant characters of capsaicin limit its percutaneous-administered dose to the low concentrations (up to $0.075 \%$ in the commercially available products) unless applied with anesthetic [10]. Capsaicin, a highly hydrophobic molecule, is conventionally administered topically in the form of ointments and gels. Most of these formulations employ organic solvents or surfactants, such as ethanol, propylene glycol and sodium lauryl sulfate as the solubilizers for capsaicin, which often disturbed or interacted with the intercellular lipids or keratin of human skin [11]. It was also reported that the increased concentrations of ethanol and isopropanol in the topical formulations reduce the percutaneous effect of capsaicin [12].

To optimize capsaicin-based topical therapy, several novel strategies have been developed to increase its local effectiveness. One strategy involved the use of capsaicin-containing patches that could be sized to fit body surface with associated pain. Recently, a new formulation, Adlea, was developed for local injection to the painful area (tissue or joint). The formulation is currently approaching the Phase III of clinical trials [13]. The only common side effect reported in the Phase I and Phase II clinical trials were initial burning pain which may be managed by premedication with the local anesthetic. Nanoencapsulated capsaicin prepared by the simple coacervation method, achieved improved bioavailability as well as covering the pungent odor usually associated with capsaicin formulations [14]. Hydroxypropyl- $\beta$-cyclodextrin complex was prepared as a mean to increase its transdermal delivery [11]. Several other novel drug delivery systems for capsaicin such as niosomes, microemulsions, lipid-polymer hybrid nanoparticles have also been developed $[15,16]$.

Lipid vesicular systems are a recognized mode of enhanced delivery of drugs into and through the skin [17]. Our particular interests were recently established ethosomes, soft, malleable vesicles tailored for enhanced delivery of active agents. Ethosomal delivery systems are mainly composed of phospholipids, ethanol and water. The phospholipid bilayers in ethosomes are packed less tightly, possess a high degree of fluidity and are reported to penetrate deeper into the skin layers, as compared to the conventional liposomes [18]. The use of phospholipid vesicles as the carrier system for capsaicin would enable its improved therapeutic effect with reduced side effects.

In an attempt to develop topical drug delivery system for capsaicin, we compared liposomes and ethosomes as carrier system in terms of both encapsulation efficiency and vesicle stability. Moreover, we directly used the crude Capsicum powder and Capsicum tincture as the sources of capsaicin. Moreover, the vesicles containing capsaicin were incorporated into the Carbopol hydrogels, to increase their stability, and prepare the skin-friendly formulation.

\section{Materials and Methods}

\section{Material}

The mature Capsicum fruits (Capsicum frutescens) were harvested from Syanja, Nepal. The shed-dried fruits were freed from seeds, grinded and stored in air tight container in the refrigerator until experiments. The Capsicum fruits were identified by comparing authentic sample and preserved in the Museum of Natural Medicine, The School of Pharmaceutical and Biomedical Sciences, Pokhara University, Pokhara, Nepal (Voucher Number 316).

All the solvents and chemicals used were of analytical grade. Methanol, acetonitrile and chloroform, glycerol and triethanolamine were the products of Merck Limited, India. Standard capsaicin (99\% purity) was purchased from Wako Pure Chemical Industries, Japan. Phosphoric acid, ammonium ferrothiocyanate and ammonium thiocyanate were the product of Qualigens Fine Chemicals, India. Lipoid S-100 (phosphatidylcholine) was a generous gift from Lipoid $\mathrm{GmbH}$, Germany. Ferric chloride hexahydrate was from S.d. Fine-Chem Ltd, India. Ethanol was purchased from Bengal Chemicals and Pharmaceutical PVT, India. We used double distilled water in all of our experiments. Dialyses tubing/ membrane (MWCO 12000-14000) was purchased from

\section{Determination of capsaicin content in Capsicum powder and Capsicum tincture}

\section{Preparation of Capsicum powder}

Seeds were removed from the shade dried Capsicum fruits and sliced fruit fragments grinded on electric stainless steel grinder. Grinding was considered to be completed when the particle size was found to be less than $200 \mu \mathrm{m}$ (determined by the sieving method). Powders were stored in an air tight container and kept refrigerated.

\section{Preparation of Capsicum tincture}

Capsicum tincture was prepared according to Japanese Pharmacopoeia XIII. In brief, one gram of Capsicum powder was accurately weighed and $10 \mathrm{ml}$ of concentrated ethanol added. The mixture was shaken on a vortex shaker for 10 minutes and finally filtered.

\section{Preparation of standard solutions}

A stock solution of capsaicin was made by dissolving $2 \mathrm{mg}$ of standard capsaicin in $2 \mathrm{ml}$ of methanol. Working solutions were prepared by diluting the stock solution to desired concentration with methanol [19].

\section{Chromatographic conditions}

HPLC system consisted of an HPLC Shimadzu pump LC-9A, a Shimadzu 6PD-6A UV spectrophotometer detector and Shimadzu C-R5A Chromatopac. Column was $\mathrm{RP}_{18}$ Fluofix 3NW415 (4.60X150 mm) joined with precolumn. The mobile 
Journal of Pharmaceutics \& Drug Development

phase consisted of $0.1 \%$ phosphoric acid: acetonitrile $(3: 2)$. The temperature of column was maintained at $30{ }^{\circ} \mathrm{C}$ during the chromatographic separation. The flow rate was $0.8 \mathrm{ml} / \mathrm{min}$ and run for 25 minutes and was monitored at UV $281 \mathrm{~nm}$.

\section{Preparation of liposomes and ethosomes}

\section{The modified film method}

Liposomes were prepared by the modified film method [20] using pure capsaicin, Capsicum tincture or Capsicum powder as a starting material to be entrapped in liposomes. Briefly, phosphatidylcholine (200 $\mathrm{mg}$ ) was dissolved in chloroform and mixed with either $5 \mathrm{mg}$ of capsaicin, $4 \mathrm{ml}$ of Capsicum tincture or $25 \mathrm{mg}$ of Capsicum powder in a round bottom flask. The solvent was evaporated in a vacuum rotary evaporator until a thin lipid film was formed on the wall of the flask. Liposomes were formed by the addition of double distilled water and hand-shaken for 10 minutes. The liposomal suspension was finally sonicated for 10 minutes in a bath sonicator. All liposomal suspensions were kept in the refrigerator prior to characterization for at least 24 hours.

\section{Preparation of ethosomes}

Ethosomes were prepared according to the procedure described by Touitou et al. [21]. Two hundred mg of Lipoid $\mathrm{S}$-100 was weighed and dissolved in $4 \mathrm{ml}$ ethanol or Capsicum tincture where applicable. Five mg of capsaicin or $25 \mathrm{mg}$ of Capsicum crude powder were added in an ethanolic solution of lipid. Under the constant stirring with magnetic stirrer, 6 $\mathrm{ml}$ of double distilled water was injected slowly and left for 30 minutes to stir under the constant stirring rate $(600 \mathrm{rpm})$. Finally, ethosomal suspension was sonicated for 10 minutes in a bath sonicator. For the preparation of ethosomes with Capsicum tincture, $4 \mathrm{ml}$ of ethanol tincture was used to dissolve $200 \mathrm{mg}$ of lipid and ethosomes prepared as previously described. All ethosomal suspensions were kept in refrigerator prior to characterization for at least 24 hours.

\section{Hydrodynamic diameter of liposomes and etho- somes}

Hydrodynamic diameter $\left(\mathrm{d}_{\mathrm{h}}\right)$ of liposomes was measured by Dynamic Light Scattering (DLS) with a vertically polarized HeNe laser beam at a wavelength of $633 \mathrm{~nm}$ (Zetasizer $3000 \mathrm{HS}$, Malvern Instruments, UK). The scattering angle was fixed at $90^{\circ}$ and temperature was maintained at $25^{\circ} \mathrm{C}$. The samples were filtered through a $1.20 \mu \mathrm{m}$ membrane prior to measurements (Chromafil $^{\circ}$, Macherey-Nagel, Germany). Analyses were performed by using the CONTIN algorithm. To assure that no vesicles were lost during pre-filtration, phospholipid content determination was performed according to the method developed by Stewart [22]. The loss of phospholipids was found to be below $1 \%(\mathrm{w} / \mathrm{w})$.

\section{Determination of entrapment efficiency for ethosomes and liposomes}

Unentrapped materials were separated from the liposomal and ethosomal suspensions by dialysis. Prior to dialysis, all samples were centrifuged at bench centrifuge to precipitate any residue of crude drug powder where applicable. Each liposomal and ethosomal sample were dialyzed against distilled water for 24 hours. The medium was continuously stirred with a magnetic stirrer. The volume of medium was adjusted to correlate with very limited solubility of capsaicin in water [23]. After dialysis, the lipids in dialyzed liposomal and ethosomal samples were destroyed by the addition of methanol, and capsaicin content was determined by the HPLC. For the ethosomes and liposomes containing ethanolic Capsicum tincture or Capsicum powder, entrapment efficiency of only active ingredient i.e. capsaicin was determined ignoring the other compounds present in the Capsicum powder and Capsicum tincture, respectively.

\section{Stability testing of liposomes and ethosomes}

The prepared liposomes and ethosomes containing capsaicin, capsaicin from Capsicum tincture or Capsicum powder, were kept at elevated temperature for the accelerated stability testing $\left(40{ }^{\circ} \mathrm{C}\right)$ over a 1 month period. Particle size and size distributions were determined prior and after one month of storage and statistically evaluated for significance ( $t$ test).

\section{Preparation of Carbopol gels}

Carbopol gels ( $1 \% \mathrm{w} / \mathrm{w})$ were prepared according to Pavelic et al. [20]. Carbopol 940 (0.250 g; Goodrich, USA) was dispersed in $22 \mathrm{~g}$ of distilled water. The mixture was stirred for 1 hour by the help of magnetic stirrer. After the thickening, glycerol $(2.5 \mathrm{~g})$ was added. Finally the formulation was neutralized by a drop-wise addition of $50 \%(\mathrm{w} / \mathrm{w})$ triethanolamine until transparent gel appeared.

Liposomal or ethosomal suspensions (freed from unentrapped material) were mixed into vehicle (gel) to produce the final concentration of liposomes/ethosomes in the gel of $15 \%$ $(\mathrm{w} / \mathrm{w})$.

\section{Evaluation of gels}

Determination of $\mathrm{pH}$ for vesicle suspensions, empty gels and gels containing vesicles was performed on $\mathrm{pH}-211$ Microprocessor $\mathrm{pH}$ Meter, Hanna Instruments, Italy.

Viscosity of gels was determined by using a viscometer (Brookfield Programmable DV2+, USA). As the starting viscosity of gel was found to be very high, each sample was diluted to a final concentration of $10 \% \mathrm{w} / \mathrm{v}$ in water and viscosity determined at $25^{\circ} \mathrm{C}$.

The gels were also evaluated visually for their appearance and integrity. The spreadability was evaluated on the upper hand skin of healthy volunteers (five).

\section{Stability studies of vesicle-based Carbopol gels}

Empty gel, liposomal and ethosomal gels were kept at $40{ }^{\circ} \mathrm{C}$ for accelerated stability testing for 3 months. The gels were evaluated microscopically at one month intervals. 


\section{Results and Discussion}

\section{Capsaicin content}

Capsaicinoids, acid amides of vanyllylamine and $\mathrm{C}_{9}-\mathrm{C}_{11}$ branched chain fatty acids, are alkaloids present in Capsicum fruit. The two major capsaicinoids present in the most varieties of Capsicum fruit are capsaicin and dihydrocapsaicin, which make up more than $91 \%$ of the total capsaicinoids. As we previously reported, the first step in standardization of crude drugs used in pharmaceutical formulations is determination of the content of active ingredient in the crude drug or drug extract [19]. As capsaicin represents the major active ingredient, we focused on the determination of capsaicin content, which was performed using the external standard method. The calibration graphs were expressed as chromatographic peak areas of standard capsaicin versus corresponding concentrations of the standard in the concentration range of 50 to $1000 \mu \mathrm{g} / \mathrm{ml}$ [21]. Calibration curve was found to be linear $(242906 \chi$-11177) with a correlation coefficient of 0.999 (Table 1). The capsaicin content in Capsicum powder was found to be $11.72 \mathrm{mg}$ per gram of powder.

\begin{tabular}{|l|l|l|l|l|}
\hline $\begin{array}{l}\text { Retention } \\
\text { time }(\mathbf{m i n})\end{array}$ & $\begin{array}{l}\text { Capsaicin } \\
\text { content }(\mathbf{m g} / \mathrm{g} \\
\text { of crude } \\
\text { powder) }\end{array}$ & $\begin{array}{l}\text { Limit of } \\
\text { detection } \\
(\mathrm{LOD} ; \\
\boldsymbol{\mu g})\end{array}$ & $\begin{array}{l}\text { Lower limit } \\
\text { of quantifica- } \\
\text { tion } \\
(\text { LOQ; } \boldsymbol{\mu g})\end{array}$ & $\begin{array}{l}\text { Correlation } \\
\text { coefficient }\end{array}$ \\
\hline 12.086 & 11.72 & 0.709 & 2.365 & 0.999 \\
\hline
\end{tabular}

Chromatographic conditions: Solvent system: $0.1 \%$ phosphoric acid-acetonitrile (3:2); Column: RP18 Fluofix 3NW415 $(4.6 \times 150 \mathrm{~mm})$ joined with precolumn; Column temperature: $30^{\circ} \mathrm{C}$; Flow rate: $0.8 \mathrm{ml} / \mathrm{min}$ and run for $25 \mathrm{~min}$; UV detection: $281 \mathrm{~nm}$; $\mathrm{LOD}=$ three times the standard error of the least square calibration curve divided by the slope

LOQ = ten times the standard error of the calibration curve divided by the slope

Table 1: Capsaicin content in capsicum powder

\section{Vesicle-based delivery system}

The incorporation of capsaicin into phospholipid vesicles is expected to increase its skin penetration, resulting in increased localized effect even at lower doses as compared with conventional creams and ointments. Liposomes and ethosomes are both considered to act as penetration enhancers. Phospholipid bilayers in ethosomes are in a more fluid state, making them more "soft" structures. The mechanism of skin permeation enhancement is proposed to be the dual fluidizing effect of ethanol on both the vesicle phospholipids as well as on the stratum corneum lipids, followed by the transport of the soft ethosomal vesicle between the disturbed stratum corneum lipid bilayers. Penetrating ethosomes also fuse with the cell membranes within the deep layers of the skin where they release their content [18]. Due to the presence of ethanol in the membrane, they provide efficient entrapment of hydrophilic, lipophilic and amphiphilic molecules. Moreover, the presence of ethanol allows for the use of standardized crude drug tinctures or extracts, such as Capsicum tincture in our case. As pure capsaicin is rather expensive, we tried to use directly the standardized Capsicum tincture or even the crude Capsicum powder as the sources of capsaicin. This is in line with the recent trend in use of standardized botanical therapeutics, such as Polyphenon E (MediGene), a topical antiviral prepared from catechins extracted from green tea (standardized mixture of active ingredients), the first such drug approved by the U.S. Food and Drug Administration in 2006 [6]. By this approach, the final cost of the formulation would be significantly reduced. Thereof, we formulated the vesicle-based delivery systems that enabled the use of the alcoholic extracts or even the crude drug powders.

In determining the entrapment efficiencies, we used the dialysis method, as it was previously reported that ultracentrifugation of ethosomes may lead to a loss of entrapped material, due to strong energy transmitted to the samples [24]. Dialysis as a separation method applied to ethosomes might also not be the optimal choice for the separation of unentrapped material as the ethanol present in the vesicles might be removed from ethosomes during dialysis. However, the entrapment values for both types of vesicles (Table 2) were rather high, in agreement with the published findings $[18,21,24]$ and much higher than for ethosomes containing for example 5-aminolevulinic acid destined for the skin administration [25].

\begin{tabular}{|l|l|l|l|l|l|}
\hline Type of vesicles & Sources of capsaicin & Capsaicin entrapment efficacy $(\%)$ & Capsaicin/Lipid ratio $(\boldsymbol{\mu g} / \mathbf{m g})$ & Size (nm) & Polydispersity index \\
\hline Liposomes & - & - & & $676.7 \pm 26.7$ & $0.65 \pm 0.03$ \\
\hline Ethosomes & - & - & & $225.2 \pm 6.1$ & $0.21 \pm 0.01$ \\
\hline Liposomes & Capsaicin & $63.83 \pm 0.24$ & 15.96 & $555.2 \pm 3.7$ & $0.44 \pm 0.03$ \\
\hline Ethosomes & Capsaicin & $56.62 \pm 5.50$ & 14.25 & $205.5 \pm 3.2$ & $0.22 \pm 0.02$ \\
\hline Liposomes & Capsicum tincture & $62.63 \pm 0.53$ & 14.66 & $215.6 \pm 5.3$ & $0.43 \pm 0.03$ \\
\hline Ethosomes & Capsicum tincture & $69.96 \pm 6.98$ & 16.38 & $150.5 \pm 3.3$ & $0.25 \pm 0.01$ \\
\hline Liposomes & Capsicum powder & $97.50 \pm 3.50$ & 1.43 & $258.9 \pm 2.4$ & $0.46 \pm 0.04$ \\
\hline Ethosomes & Capsicum powder & $88.57 \pm 3.60$ & 1.30 & $129.1 \pm 3.9$ & $0.24 \pm 0.02$ \\
\hline
\end{tabular}

Liposomes prepared by the modified film method and ethosomes prepared according to Touitou et al. (2000) were made of phosphatidylcholine (200 mg; Lipoid S-100) and either pure capsaicin (5 mg), Capsicum tincture ( $4 \mathrm{ml}$ ) or crude Capsicum powder $(25 \mathrm{mg})$

Table 2: Characterization of vesicles $(n=3)$ 
Journal of Pharmaceutics \& Drug Development

The size of prepared vesicles was appropriate for topical administration and did not significantly increase during the accelerated stability study $\left(40^{\circ} \mathrm{C}\right.$; Figure 1$)$. The vesicle size remained similar during the accelerated testing for both types of vesicles (Figure 1) and corresponded to the size of ethosomes reported in reference [26]. Godin and Touitou [27] tested the stability of ethosomes over 1-year storage at room temperature and found that the size of the ethosomal suspensions did not change significantly. Dubey et al. [28] observed the release of entrapped melatonin at increased temperature.

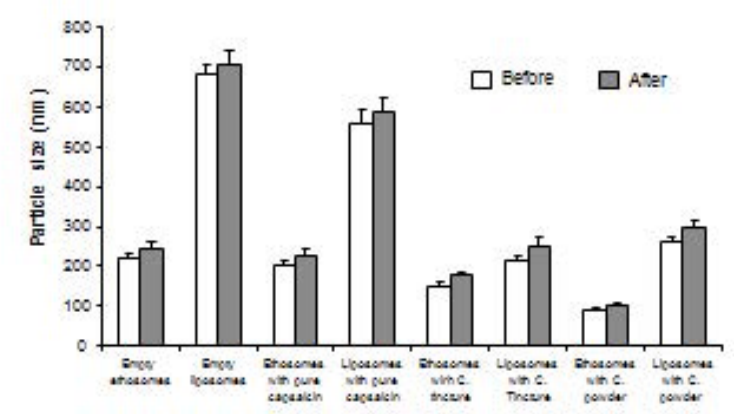

Legend: Stability was observed by measuring particle size after 1 month at 40 ${ }^{\circ} \mathrm{C}$. No significant increase in average vesicle size was observed during accelerated stability testing. The values represent the mean \pm SD of three separate experiments (duplicate). (See Experimental part for details)

Figure 1: Vesicle stability expressed as an increase in particles size during 1 month storage at $40^{\circ} \mathrm{C}$

Several reports dealing with ethosome stability and increase in the size indicate that ethosomes may become leaky, such as in the case of ketotifen-containing ethosomes [29]. However, one has to note that ketotifen is a drug with surface activeproperties and may destabilize the membranes causing the leakage. On the other hand, ethosomes with methotrexate were reported to be leaky at increased temperature during 120 days of storage, while the size did not change significantly [30]. Other reported that the vesicle size remained unchanged for 4 weeks storage at room temperature [24]. Ethosomes stored at refrigerated conditions $\left(4^{\circ} \mathrm{C}\right)$ remained substantially stable, both in terms of drug entrapment and particle size during the storage for 3 months [31].

To increase the viscosity of topical formulation and manufacture a more skin-friendly formulation, we further incorporated vesicles into the Carbopol hydrogels. In this way the original structure and the integrity of vesicles are expected to remain stable [20].

\section{Gels as vehicle for vesicle-based drug delivery systems}

Gels, particularly hydrogels, are considered to be superior vehicle in topical administration of vesicular suspensions $[20,32,33]$. Moreover, hydrogels are known to provide a faster release of lipophilic drugs and a high clarity in appearance, which makes them an attractive topical dosage form. Carbopol gels exhibit good fluxes and accumulative amounts of drugs [12]. The hydrogel base exhibits potent hydrophilic properties and Carbopol hydrogels are reported to increase the permeation of capsaicin [34]. However, the amount of
Carbopol in a hydrogel should be kept low, not to affect the penetration flux through the skin as it was reported that the flux of capsaicin increased with the decrease in the Carbopol

In vitro permeation of capsaicin from hydrogels depends on the physicochemical nature and the concentration of the polymer used [34]. For hydrogels containing liposomes, the membrane rigidity and lipid concentrations are known to influence the integrity of liposomes dispersed in gels [35]. For the hydrophilic drugs, liposomes containing less rigid membranes provide faster release, whereas in the case of lipophilic drugs, the lipophilicity of a molecule and the lipid concentration are the predominant factors [36].

The viscosity of hydrogels is one of the main features in determining their applicability and patient acceptance of topical formulations. Considering that the formulation should be applied to the painful area, its spreadability directly affects overall evaluation of the formulation.

The $\mathrm{pH}$ of prepared hydrogels was ranging from 6.41 to 7.17 (Table 3), which was found to be acceptable and close to the $\mathrm{pH}$ of the skin. Ethosomal gel exhibited higher $\mathrm{pH}$ as a result of the presence of ethosomal suspensions exhibiting higher $\mathrm{pH}$ than the liposomal ones. Neutral $\mathrm{pH}$ was found to provide the best consistency of gel. The highest viscosity was observed for the gels neutralized to $\mathrm{pH}$ 6. As it is known that buffers may affect the viscosity of hydrogels [20], we prepared all vesicle suspensions and hydrogels in a double distilled water to avoid the effect of buffer ions on gel viscosity. After the three months storage at the accelerated conditions, no visual changes in the appearance, as determined microscopically, were observed (Table 4). The spreadability of gels was very satisfactory and the appearance was of an attractive skin-friendly formulation. We are aware that the visco-elastic measurements or more sophisticated rheological devices would provide further insight on the influence of ethosomal suspensions on the rheological properties of hydrogels and would give deeper insight on the actual interaction between those vesicles and the hydrogels. However, since no changes in the appearance were visualized, we assumed that the system was rather stable.

The only reported liposomal capsaicin up to now was destined for intravesical therapy of detrusor hyperreflexia, where liposomal capsaicin has been proven to be superior to hydrogels and $30 \%$ ethanolic solutions containing the same active ingredient at the same concentration [37].

\begin{tabular}{|c|c|c|}
\hline Sample & pH (mean \pm S.E. $)$ & Viscosity $(\mathrm{cPs})^{*}$ \\
\hline Empty gel & $6.41 \pm 0.01$ & $2650 \pm 97$ \\
\hline Liposomal gel & $6.37 \pm 0.03$ & $1850 \pm 119$ \\
\hline Ethosomal gel & $7.17 \pm 0.05$ & $3517 \pm 123$ \\
\hline Gel containing ethanol ${ }^{*}$ & $6.89 \pm 0.05$ & nd \\
\hline Liposomal suspension & $6.12 \pm 0.03$ & nd \\
\hline Ethosomal suspension & $7.67 \pm 0.02$ & nd \\
\hline
\end{tabular}

Gel samples were diluted to $10 \%(\mathrm{w} / \mathrm{v})$ prior to the viscosity measurements.

*Vesicles-free gel was prepared with the same amount of ethanol as contained in the ethosomal gel.

Table 3: Characterization of ethosomal and liposomal hydrogels $(n=3)$ 


\begin{tabular}{|l|l|}
\hline Type of gel & Evaluation \\
\hline Empty gel & No change \\
\hline Gel containing ethanol ${ }^{*}$ & No change \\
\hline Liposomal gel & No change \\
\hline Ethosomal gel & No change \\
\hline
\end{tabular}

All samples were kept at $40{ }^{\circ} \mathrm{C}$ and microscopically examined every month during 3 months period. The consistency of gel was compared to the consistency at day 0 .

${ }^{\star}$ Vesicles-free gel was prepared with the same amount of ethanol as contained in ethosomal gel.

Table 4: Accelerated stability of ethosomal and liposomal hydrogels

\section{Conclusions}

To the best of our knowledge, it is the first paper reporting the vesicular formulations for capsaicin destined for localized pain relieve based on the use of the crude Capsicum powder and Capsicum tincture, instead of pure capsaicin. Our findings implement that costly and time-consuming process of capsaicin isolation may be avoided, resulting in a rather straight-forward formulation development.

Moreover, the high entrapment efficiencies indicate that the need to remove the unentrapped material prior to vesicle incorporation into hydrogels may be neglected, reducing the time and final cost of the formulation. Both liposomal and ethosomal hydrogels have a potential to increase capsaicintreatment efficiency and patient compliance.

\section{Acknowledgment}

The continuous generosity of Lipoid GmbH, Ludwigshafen, Germany, is highly acknowledged.

\section{References}

1. Kesarwani K, Gupta R, Mukerjee A (2013) Bioavailability enhancers of heral origin: An overview. Asian Pac J Trop Biomed 3: 253-266.

2. Wieland HA, Michaelis M, Kirschbaum BJ, Rudolphi KA (2005) Osteoarthritis-an untreatable disease? Nature Rev. Drug Discovery, 4: 331-345.

3. Knotkova H, Pappagallo M, Szallasi A (2008) Capsaicin (TRPV1 agonist) therapy for pain relief, Farewell or revival. Clin J Pain, 24: 142-154.

4. Treede RD, Wagner T, Kern KU, Husstedt IW, Arendt G (2013) Mechanism- and experience-based strategies to optimize treatment response to the capsaicin $8 \%$ cutaneous patch in patients with localized neuropathic pain. Curr Med Res Opinion 29: 527-538.

5. Moore RA, Derry S, McQuay HJ. (2008) Topical agents in the treatment of rheumatic pain. Rheum Dis Clin North Am, 34: 415-432.

6. Corson TW, Crews CM (2007) Molecular understanding and modern application of traditional medicines: Triumphs and Trials Cell 130: 769-774.

7. Mason L, Moore RA, Derry S, Edwards JE, McQuay HJ (2004) Systematic review of topical capsaicin for the treatment of chronic pain. BMJ 328: 991.

8. Pershing LK, Reilly CA, Corlett JL, Crouch DJ (2004) Effects of vehicle on the uptake and elimination kinetics of capsaicinoids in human skin in vivo. Tox Appl Pharm 200: 73-81.

9. Chanda S, Bashir M, Babar S, Koganti A, Bley K (2008) In vitro hepatic and skin metabolism of capsaicin. Drug Metab Dispos 36: 670-675.

10. Fang JY, Wu PC, Huang YB, Tsai YH. (1996). In vivo percutaneous absorption of capsaicin, nonivamide and sodium nonivamide acetate from ointment bases: Pharmacokinetic analysis in rabbits. Int J Pharm 128: 169-177.

11. Zi P, Yang X, Kuang H, Yang Y, Yu L (2008) Effect of HPbetaCD on solubility and transdermal delivery of capsaicin through rat skin. Int J Pharm 358: 151-158.

12. Fang JY, Wu PC, Huang YB, Tsai YH (1996) Percutaneous absorption of capsaicin, nonivamide and sodium nonivamide acetate from gel and ointment bases; in vitro formulation evaluations in pigs and in vivo bioengineering methods in humans. Int J Pharm 130: 121-135.

13. Remadevi R, Szallasi A (2008) Adlea (ALGRX-4975), an injectable capsaicin (TRPV1 receptor agonist) formulation for long-lasting pain relief. IDrugs 11: 120-132.

14. Wang JC, Chen SH, Xu ZC (2008) Synthesis and properties research on the nanocapsulated capsaicin by simple coacervation method. J Dispersion Sci Tech 29: 687-695.

15. Tavano L, Alfano P, Muzzalupo R, de Cindio B (2011) Niosomes vs microemulsions: New carriers for topical delivery of capsaicin. Colloid Surf B: Biointerfaces 87: 333-339.

16. Desai PR, Marepally S, Patel AR, Voshavar C, Chaudhuri A, et al. (2013) Topical delivery of anti-TNFa siRNA and capsaicin via novel lipid-polymer hybrid nanoparticles efficiently inhibits skin inflammation in vivo. J Control Release 170: 51-63.

17. El Maghraby GM, Barry BW, Williams AC (2008) Liposomes and skin: From drug delivery to model membranes. Eur J Pharm Sci 34: 203-222.

18. Elka T, Biana G (2007). Dermal drug delivery with ethosomes: therapeutic potential. Therapy 4: 465-472.

19. Thapa B, Skalko-Basnet N, Takano A, Masuda K, Basnet P (2009) HPLC analysis of capsaicin content in sixteen Capsicum fruits from Nepal. J Med Food 12: 908-913.

20. Pavelic Z, Skalko-Basnet N, Schubert R (2001) Liposomal gels for vaginal drug delivery. Int J Pharm 219: 139-149.

21. Touitou E, Dayan N, Bergelson L, Godin B, Eliaz M (2000) Ethosomes - novel vesicular carriers for enhanced delivery: characterization and skin penetration properties. J Control Release 65: 403-418.

22. Stewart JCM (1980) Colorimetric determination of phospholipids with ammonium ferrothiocyanate. Anal Biochem 104: 10-14.

23. Kasting GB, Fransis WR, Bowman LA, Kinnett OG (1997) Percutaneous absoprtion of vanilloids: In vivo and in vitro studies. J Pharm Sci 86: 142-146.

24. Lopez-Pinto JM, Gonzalez-Rodriguez ML, Rabasco AM (2005) Effect of cholesterol and ethanol on dermal delivery from DPPC liposomes. Int J Pharm 298: 1-12.

25. Fang YP, Huang YB, Wu PC, Tsai YH (2009) Topical delivery of 5-aminolevulinic acid-encapsulated ethosomes in a hyperproliferative skin animal model using the CLSM technique to evaluate the penetration behaviour. E J Pharm Biopharm 73: 391-398.

26. Paolino D, Lucania G, Mardente D, Alhaique F, Fresta M (2005) Ethosomes for skin delivery of ammonium glycyrrhizinate: In vitro percutanous permeation through human skin and in vivo anti-inflammatory activity on human volunteers. J Cont Rel 106: 99-110.

27. Godin B, Touitou E (2005) Erythromycin ethosomal systems: Physicochemical characterization and enhanced antibacterial activity. Cur Drug Deliv 2: 269-275.

28. Dubey V, Mishra D, Jain NK (2007) Melatonin loaded ethanolic liposomes: Physicochemical characterization and enhanced transdermal delivery. Eur J Pharm Biopharm 67: 398-405.

29. Elsayed MMA, Abdallah OY, Naggar VF, Khalafallah NM (2007) Deformable liposomes and ethosomes as carriers for skin delivery of ketotifen. Pharmazie 62: 133-137.

30. Dubey V, Mishra D, Dutta T, Nahar M, Saraf DK, et al. (2007b) Dermal and transdermal delivery of an anti-psoriatic agent via ethanolic liposomes. J Cont Rel 123: 148-154.

31. Maestrelli F, Capasso G, Gonzales-Rodriguez ML, Rabasco AM, Ghelardini C, et al. (2009) Effect of preparation technique on teh properties and in vivo efficacy of benzocaine-loaded ethosomes. J Liposome Res 19: 253-260. 
32. Skalko N, Cajkovac M, Jalsenjak I (1998) Liposomes with metronidazole for topical use: The choice of preparation method and vehicle. J Lipos Res 8: 283-293.

33. Hurler J, Berg OA, Skar M, Conradi AH, Johnsen PJ, et al. (2012) Improved burns therapy: Liposomes-in-hydrogel delivery system for mupirocin. J Pharm Sci 101: 3906-3915.

34. Wang YY, Hong CT, Chiu WT, Fang JY (2001) In vitro and in vivo evaluations of topically applied capsaicin and nonivamide from hydrogels. Int J Pharm 224: 89-104.
35. Mourtas S, Haikou M, Theodoropoulou M, Tsakiroglou C, Antimisiaris SG (2008) The effect of added liposomes on the rheological properties of a hydrogel: A systematic study. J. Colloid Interf Sci 317: 611-619.

36. Mourtas S, Fotopoulou S, Duraj S, Sfika V, Tsakiroglou C, et al. (2007) Liposomal drugs dispersed in hydrogels, Effect of liposome, drug and gel properties on drug release kinetics. Colloids Surf B: Biointerf 55: 212-221.

37. Tyagi P, Chancellor MB, Li Z, De Groat WC, Yoshimura N, et al. (2004) Urodynamic and immunohistochemical evaluation of intravesical capsaicin delivery using thermosensitive hydrogel and liposomes. J Urol 171: 483-489. 